\title{
MORPHING WING APPLICATION ON HYDRA TECHNOLOGIES UAS-S4
}

\author{
Marine Segui ${ }^{1}$, Oliviu Sugar Gabor ${ }^{2}$ Andreea Koreanschi ${ }^{3}$ and Ruxandra Mihaela Botez ${ }^{4}$ \\ ETS, Laboratory of Active Controls, Avionics and AeroServoElasticity LARCASE \\ 1100 Notre Dame West, Montreal, Quebec, Canada, H3C-1K3
}

\begin{abstract}
This paper presents the aerodynamic results of a morphing wing study performed on the UAS S4 Éhecatl from Hydra Technologies. Only the cruise phase of the aircraft was considered (constant altitude and constant speed). The difference, from an aerodynamic point of view, between the morphing wing and the original wing was emphasized by computing and comparing their longitudinal aerodynamic coefficients (drag and lift). The computation of the aerodynamic characteristics was done using tornado with the Vortex Lattice Method.
\end{abstract}

\section{KEY WORDS}

Unmanned Aerial System, aerodynamic optimization, Morphing Wing.

\section{Introduction}

The globalization has made that people travel increasingly by air transportation. Meanwhile, some environmental conferences are organized against the global warming to regulate carbon dioxide (CO2) emissions as result of new society consumption. In the aeronautic industry, a means to reduce carbon dioxide emission have to be finding. Since 1998, Air Force Research Laboratory (AFRL), FlexSys Inc and NASA's test team are working together to reduce fuel cost. They suggest to modify flight control surfaces on existing aircraft such as flaps in Adaptive Compliant Trailing Edge (ACTE) project [1]. Based on this idea, some principles were developed to improve carbon dioxide emission, above all, a special material [2] or a smart wing project $[3,4]$ nowadays called "morphing wing".

In this paper the morphing wing technology consists in the change of the wing's airfoil shape during flight [5]. This concept, showed improved the performances of the wing's airfoil through optimization of the original airfoil; the optimization was carried at different velocities, angles of attack and Reynolds numbers. The drag was reduced, while the lift was increase or kept constant (depend on case) at all flight phases [6-9].

The aerodynamic study here presented consists in applying the morphing wing's airfoil concept on a part of span wing, and ensuring the improvement of the wing's aerodynamic performances.
In order to study impacts of morphing wing technology an aerodynamic comparison of morphed and un-morphed (original) wing is used. The comparison can be done by longitudinal aerodynamics coefficient computation [10] from equations of longitudinal aircraft motion [11]. To test the morphing wing effects, an available aircraft such as the UAS S4 of Hydra Technology (Figure 1) was selected. Using an UAV when studying and applying state of the art research has several benefits: low costs in its design, validation using wind tunnel and in flights tests, or a faster certification.

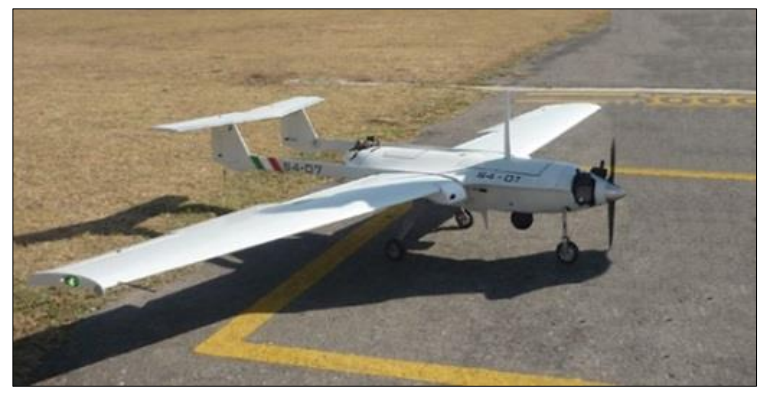

Figure 1: Hydra Technology UAS-S4 Unmanned Aerial System

\section{Aerodynamic Coefficients Computation}

The following methodology computes wing aerodynamic coefficients from geometry data. The original wing considered is carried by UAS-S4 Ehécatl. In this paper this wing is called "Hydra". A constant sweep angle with the same airfoil from the root to the tip are the particularities of this wing.

\subsection{Hydra Technology UAS-S4 Ehécatl}

The UAS-S4 Ehécatl (Figure 1) is a military Unmanned Aerial Vehicle (UAV). It is used for overseeing operations on a given range. This range depends particularly on the geometrical characteristics (Figure 2), the maximum weight available (fuel and equipment), and the propeller engine power. 


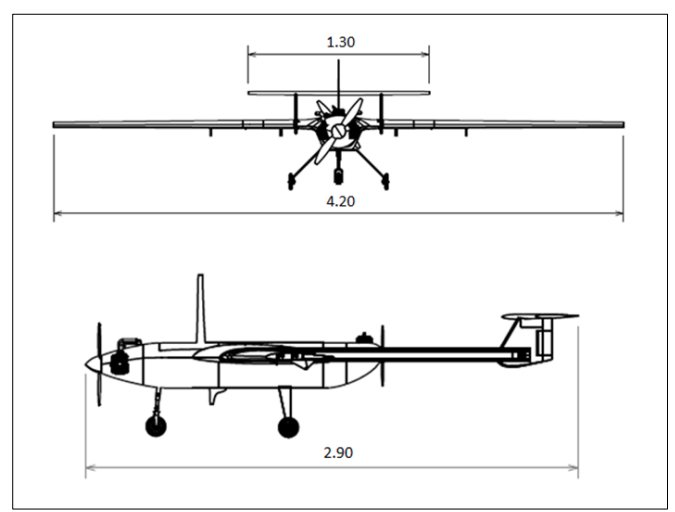

Figure 2: UAS S4 plane

The maximum takeoff weight for this UAV is $80 \mathrm{~kg}$ including approximately $45 \mathrm{~kg}$ of equipment. A push-pull configuration propels the UAV at a maximum altitude of $15000 \mathrm{ft}$ for a maximum Mach number of 0.18 . At these flight conditions, the flow is considered laminar, as it is considered if there were low Reynolds numbers. This hypothesis will enable simplifications for some computations methods such as ANSYS Fluent with the k$\omega$ SST turbulence model coupled with the $\gamma-\operatorname{Re}_{\theta}$ model, Tornado with Prandtl theory, or XFLR5.

\subsection{Application of the Morphing Wing Concept on UAS-S4}

The morphing wing technology use an internal mechanism equipped with electrical or SMA actuators allowed the modification of the upper surface of the wing airfoil depending on flight conditions (speed, angle of attack or aileron deflection). The aim of the mechanism (Figure 3) was to deform the upper surface of the airfoil in order to determine a positive change in the aerodynamic parameters of lift and drag, depending on the desired purpose; e.g. increase lift, decreased drag or combinations. The actuators change the airfoil shape during the flight by means of a PID controller [12-14].

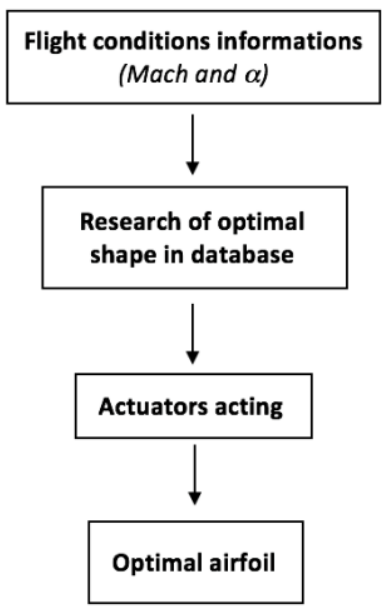

Figure 3: Morphing Wing System methodology
The Research Laboratory in Active Controls, Avionics and Aeroservoelasticity (LARCASE), at "École de technologie superieure" (ETS) has developed state-ofthe-art research in the domain of 'Morphing Wing' during in the CRIAQ 7.1 and the CRIAQ MDO 505 wing projects $[7,9,15]$. The Morphing Wing concept presented in Figure 3 was successfully applied in the CRIAQ MDO 505 project. In this project, the upper surface of the wing, between $20 \%$ and $65 \%$ of the chord, was replaced with a flexible composite skin [10]. New shapes of the upper surface region were determined through optimization of the local wing airfoil at various flight conditions (speed, angle of attack and aileron deflection) The wind tunnel experiments performed on the active morphing wing mode have shown improvement in the behavior of the boundary layer, by delaying the transition from the laminar to turbulent state, which indirectly determines a decrease in the drag coefficient, without modifying the lift values.

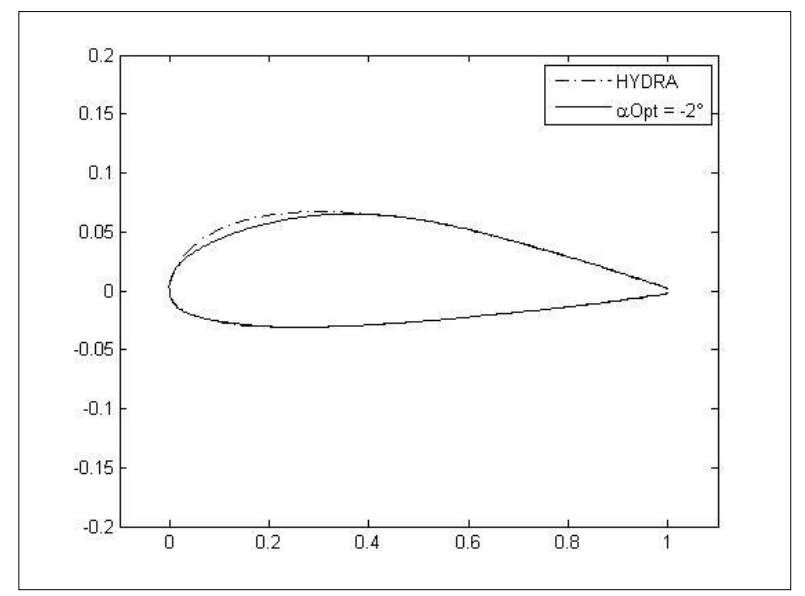

Figure 4: Morphed airfoil for $M=0.15$ and $\alpha=-2^{\circ}$

Based on the research performed during the CRIAQ MDO 505, the morphing concept was applied differently for the wing of the UAS-S4. For the UAS-S4, the wing has to change its shape between $20 \%$ of the chord on its inner surface and $65 \%$ of the chord on its upper surface. For the research presented in this paper, optimized airfoils were obtained for several flight cases: Mach number 0.15 and angles of attack between $-3^{\circ}$ to $+3^{\circ}$. The new airfoil shapes were obtained using an in-house developed optimization algorithm coupled with Xfoil aerodynamic solver for estimation of the lift and drag performance of the airfoils $[16,17]$. Figure 4 presents an example of the shape optimization results. It shows the original airfoil "Hydra" and the morphed optimized airfoil for Mach number 0.15 and an angle of attack $\alpha=-2^{\circ}$ called " $\alpha \mathrm{Opt}$ $2^{\circ}$ ". 


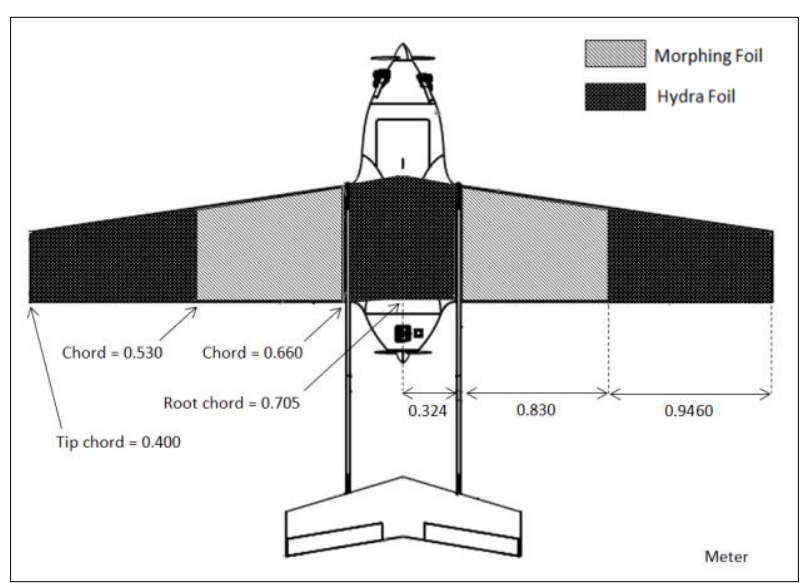

Figure 5: Optimized and original airfoil combination

In this paper it is proposed to reconstruct the geometry of the UAS-S4 wing using the optimized airfoil shapes obtained for the flight cases mentioned before. The morphed wing is a combination of the original airfoil and optimized airfoil such is defined in Figure 5. Therefore, because the optimized airfoil depends on the Mach number and the angle of attack, the wing will dynamically change its shape during flight at Mach 0.15 and passing through each angle of attack between $-3^{\circ}$ to $+3^{\circ}$.

\subsection{Longitudinal aerodynamics coefficients}

In this section, the methodology for calculating the lift and drag coefficients was presented. The lift and the drag forces are given in equations (1) and (2).

$$
\begin{aligned}
& L=\frac{1}{2} * \rho * S * V^{2} * C_{L} \\
& D=\frac{1}{2} * \rho * S * V^{2} * C_{D}
\end{aligned}
$$

Where $\rho$ is the density of air flow, $S$ is the area of the wing (assumed to be the same for the morphing wing), and $V$ is the true airspeed. Aerodynamic studies can be performed for these lift and drag coefficients.

$$
C_{L}=C_{L \alpha} . \alpha+C_{L_{0}}
$$

The lift coefficient given by equation (3) can be separated into two parts. The first part is induced by the angle of attack derivative $C_{L \alpha}=2 \pi * \alpha$ where $\alpha$ is the angle of attack in radians, while the second part, $C_{L O}$ is computed from the airfoil lift characteristics at zero angle of attack.

$$
C_{D}=C_{d 0}+\frac{C l^{2}}{\pi \cdot A R \cdot e}
$$

The drag coefficient is also composed by two parts, as shown in equation (4), where $C_{d 0}$ is the parasite drag, $C l$ is the lift coefficient for an airfoil, $A R$ is the aspect ratio $\left(\mathrm{AR}=\mathrm{b} / \mathrm{S}^{2}\right), \mathrm{b}$ is the wing span and $e$ is the efficiency factor.

\subsection{Computation Methods}

Several methods permit the computation of wing aerodynamics coefficients [18-20]. In literature, the aerodynamics methods that were often used were implemented in the ANSYS Fluent and Tornado software [10].

\subsubsection{Computational Fluid Dynamics Method}

A reliable commercial aerodynamic computation is supported by ANSYS Fluent software. ANSYS Fluent computed pressure applied on a three dimensions subject by means of Computational Fluid Dynamics (CFD) method. The subject and it environment have to be meshed according to the precision of the calculation. The fluid pressure on each part of mesh is solved by projections of fluid dynamics equations. Pressure of the entire subject is obtained by an iterative calculation on the three dimensions subject. To obtain reliable results, the mesh has to be small enough. An interesting mesh takes in consideration all the details of the subject. In this study, all deformations induced by actuators have to be very well considerate so a refined mesh is needed even if it induced a long time of computation. To reduce operating mistake the mesh was generated by ANSYS ICEM CFD software. The mesh considerate here (Figure 6) is the same that was used for MDO 505 wing project [9], the wing is defined by 400 cells around the airfoil and 160 cells along the span. ANSYS Fluent is one of the most known software commercially available to compute aerodynamics coefficients.

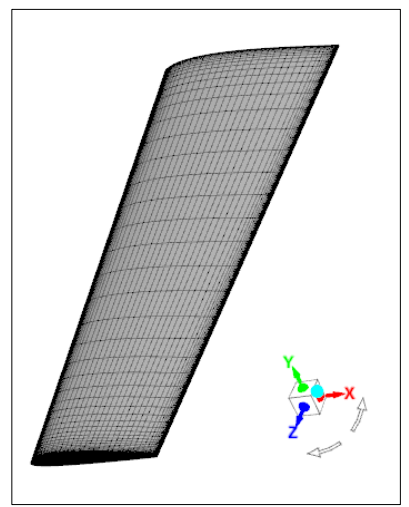

Figure 6: Semi-Wing span on Fluent

The disadvantage of this software is that it uses long computation time to calculate the aerodynamic coefficients for one flight condition (one angle of attack and one Mach value). Although it is recognized that the ANSYS Fluent software gives very good approximations of the aerodynamics coefficients [21], when working with a large number of cases, a faster method, such as those implemented in the Tornado software, was needed. 


\subsubsection{Vortex Lattice Method}

The Vortex Lattice Method (VLM) is a method dedicated to compute aerodynamic coefficients of wings. In 1966, in the early years of these method, only thin wings were considered in the VLM [22] but that hypothesis was excessively large. Then in 1993, the VLM was improved in order to consider more precisely the components of the wing, with the nonlinear motions of flaps [23] for example. Nowadays, small details of the wing geometry need to be taken into account in order to improve the quality of the computational results. The VLM method that was used in this paper, was implemented in the Tornado platform, run by Matlab software [24]. Therefore, the wing is entirely modelled according to the three dimensions (3D) panels method, the geometry of the wing considered by the method can be showing like Figure 7 (two dimensions), and an airfoil is associated to this wing, which represent the third dimension. If the wing is not carried by the same airfoil from the root to the tip (original airfoil such as Hydra), Tornado platform allow to "cut" the two dimensions of the wing in the span to create wing sections, called "semi-wing". In this case, a different airfoil can be assigned to each "semi-wing", actually, it's the procedure when the morphing wing is computed. For the UAS-S4, three symmetrical semi wing were create following Figure 5, original airfoil is assigned around the root and around the tip sections of the wing and Optimized airfoil is assigned in the middle part.

The flow was considered to be described by the Mach number (altitude and speed parameters).

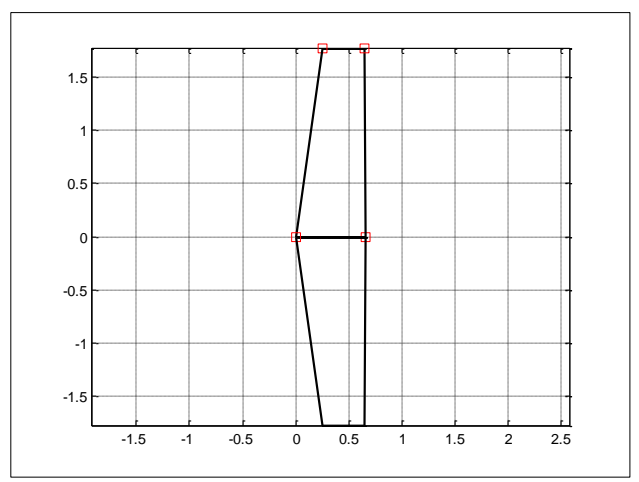

Figure 7: Wing definition in the Tornado software

Although Tornado software is very interesting from the point of view of the rapidity of the computation time (around 10 seconds per airfoil for 13 angles of attack), this method needed many approximations of the wing modeling. To ensure Tornado software make reliable approximations, a study was made to confirm this method. In this respect, a comparison was made between aerodynamic coefficients obtained by Tornado software and ANSYS Fluent software (Figure 8 and Figure 9). The wing considered carried by Hydra airfoil from the root to the tip.

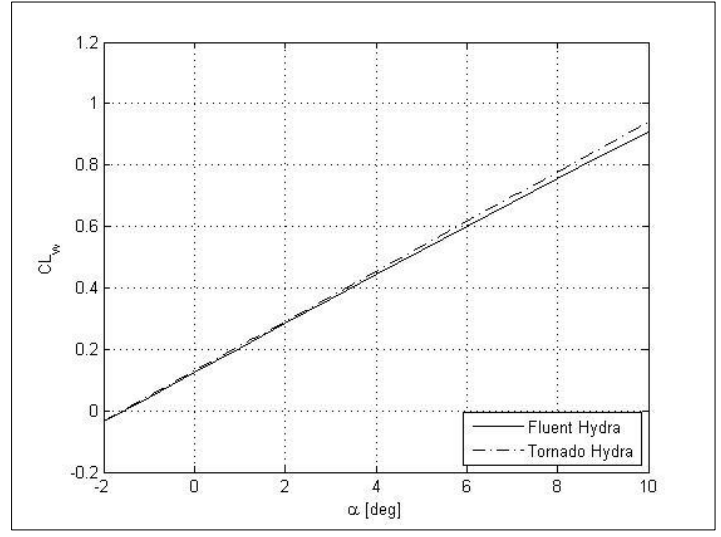

Figure 8: Variation of lift coefficients with the angle of attack computed by ANSYS Fluent and Tornado software for Mach number 0.15

Figure 8 represents the lift coefficient variation for several angles of attack values from -2 degrees to 10 degrees. In this Figure, the lift coefficients computed by Tornado fits very well the lift coefficient curve computed by ANSYS at low angles of attack, less than $3^{\circ}$. The error does not exceed $3 \%$ for angles of attack below +3 degrees as shown on Table 1. Because each morphed airfoil were optimized for an angle of attack between -3 degrees to +3 degrees, the morphing wing is restricted to this range. Accordingly to comments of Figure 8, Tornado software is validating for lift coefficient computation.

\begin{tabular}{|c|c|c|c|}
\hline$\alpha\left[^{\circ}\right]$ & $\mathrm{C}_{\mathrm{L}}$ ANSYS Fluent & $\mathrm{C}_{\mathrm{L}}$ Tornado & \% error \\
\hline-2 & -0.0337 & -0.0338 & -0.34 \\
\hline 0 & 0.1248 & 0.1284 & -2.86 \\
\hline 2 & 0.2841 & 0.2907 & -2.32 \\
\hline 4 & 0.4435 & 0.4530 & -2.16 \\
\hline 6 & 0.6015 & 0.6154 & -2.31 \\
\hline 8 & 0.7565 & 0.7777 & -2.79 \\
\hline 10 & 0.9058 & 0.9400 & -3.77 \\
\hline
\end{tabular}

Table 1: Lift coefficients values computed by ANSYS Fluent and Tornado for Mach number 0.15 and 7 angles of attack

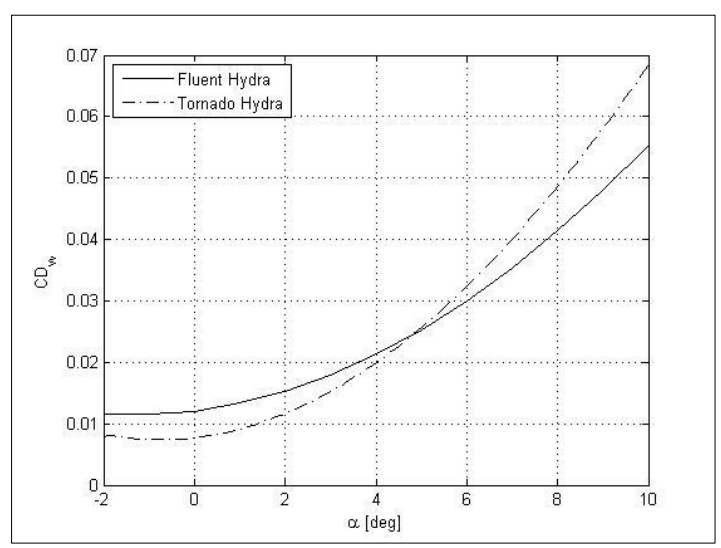

Figure 9: Variation of drag coefficients with the angle of attack computed by ANSYS Fluent and Tornado software for Mach number 0.15 
Figure 9 represents the drag coefficients variation with $\alpha$ computed by ANSYS Fluent and Tornado for the same angles of attack values as ones considered for the lift coefficients variation with $\alpha$. A small dissimilarity difference between results can be seen in Figure 9. Table 2 shows the relative errors calculated for the drag coefficients. A maximum of $36 \%$ of error was found. For the purpose of determining the level of improvement for the morphing wing, a relative error if $30 \%$ was considered acceptable. And that the difference comes for the methods used by the two solvers, which use different approaches in calculating the drag force. Although the relative error of $30 \%$, Tornado software is also validating for drag coefficient computation.

\begin{tabular}{|c|c|c|c|}
\hline$\left.\alpha{ }^{\circ}\right]$ & $\begin{array}{c}\mathrm{C}_{\mathrm{D}} \text { ANSYS } \\
\text { Fluent }\end{array}$ & $\mathrm{C}_{\mathrm{D}}$ Tornado & $\%$ error \\
\hline-2 & 0.0116 & 0.0078 & 29.85 \\
\hline 0 & 0.0120 & 0.0077 & 36.00 \\
\hline 2 & 0.0153 & 0.0116 & 27.17 \\
\hline 4 & 0.0212 & 0.0199 & 10.92 \\
\hline 6 & 0.0300 & 0.0323 & -3.18 \\
\hline 8 & 0.0413 & 0.0486 & -13.39 \\
\hline 10 & 0.0553 & 0.0684 & -19.96 \\
\hline
\end{tabular}

Table 2: Drag coefficients computed by ANSYS Fluent and Tornado software for Mach number 0.15

\section{Results}

The $C_{L}$ and $C_{D}$ variations with $\alpha$, the aerodynamics polar and the finesse ratio variation with $\alpha$ are presented in this section. These results were obtained using the Tornado software. The morphing wing is represented with a curve because it's considered that the airfoil move its shape continuously between two angles of attack.

\subsection{Lift and Drag improvement}

The variations of lift and drag coefficients with $\alpha$ are presented in Figure 10. The relative error observed between the lift coefficient computed for the morphing wing and the original wing is approximately $2 \%$ for negative angles of attack and $0.5 \%$ for positives angles of attack. Thus, the improvement on lift of the morphing airfoil is small. As an improvement of the lift coefficient was not part of the optimization objective, these results are considered as a sufficiently small difference and the objective of maintaining the lift constant was considered achieved.
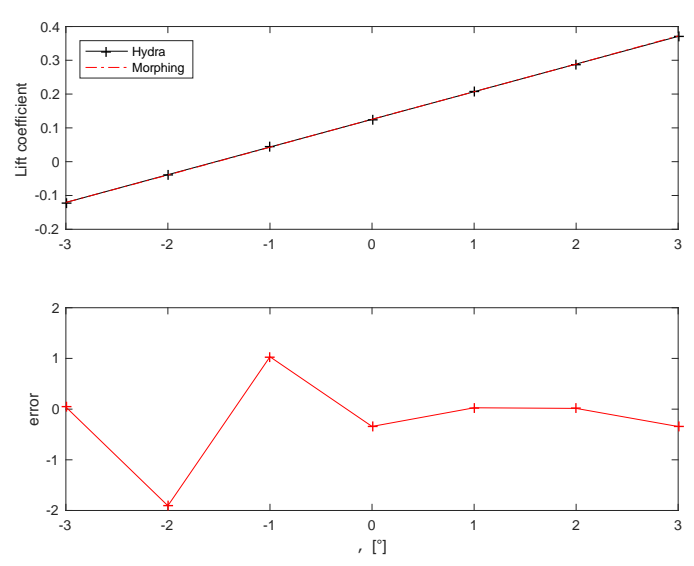

Figure 10: Lift coefficient variation with the angle of attack for the Hydra wing and the morphing wing and for Mach number 0.15

The relative error obtained for the drag coefficient variation with $\alpha$ (Figure 11) is the most representative of the morphing wing improvement, as this represent the optimization objective. For all of $\alpha$ value the drag coefficient of morphing wing is below the drag coefficient of the original wing. As mentioned in section 2.3, because of the fact that the drag acts as a friction force, if it's reduced, then an aerodynamics improvement is found for the morphing wing. The Figure 11 also shows that the relative error was around $1 \%$ for all angles of attack.

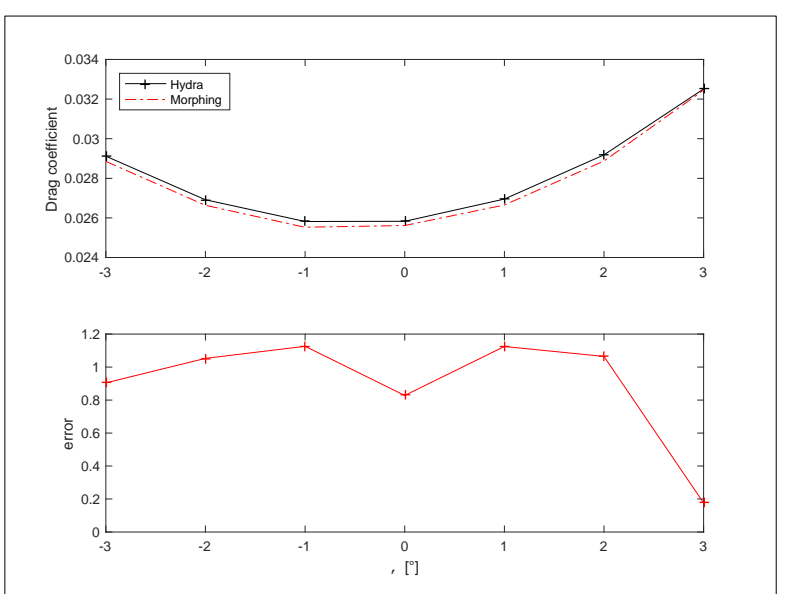

Figure 11: Drag coefficient variation with angles of attack for the Hydra wing and the morphing wing for Mach number 0.15

Within this context, morphing wing has on the whole, higher performances than the reference wing.

\subsection{Aerodynamics parameters improvement}

To give a general conclusion on morphing wing performance, some aerodynamics tools allow to compare wings performance, especially the aerodynamic polar and the lift over drag ratio, as shown in the following subsections. 


\subsubsection{The aerodynamic polar}

The aerodynamic performances of a wing are expressed in terms of the best lift over drag ratio.

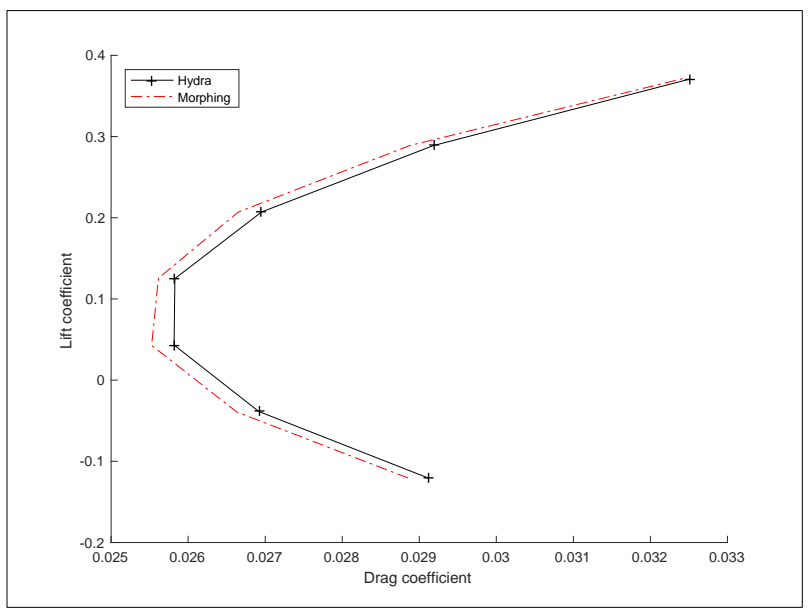

Figure 12: Aerodynamics polar comparison between the Hydra wing and the morphing wing for Mach number

0.15

Figure 12 shows the aerodynamic polar comparison for the two wings airfoils. "Morphing" curve corresponds to the morphing wing while "Hydra" curve corresponds to the original fixed wing. A first observation shows that the morphing curve is translating to the left of Hydra curve. As mentioned the lift experienced a very slight increase while the drag decreased. Since no translations up-down can be observed, therefore, no improvement was found for the lift coefficient. Generally speaking, the morphing of the reference wing improved the aerodynamics polar of the Hydra's wing.

\subsubsection{Lift over drag (L/D) ratio}

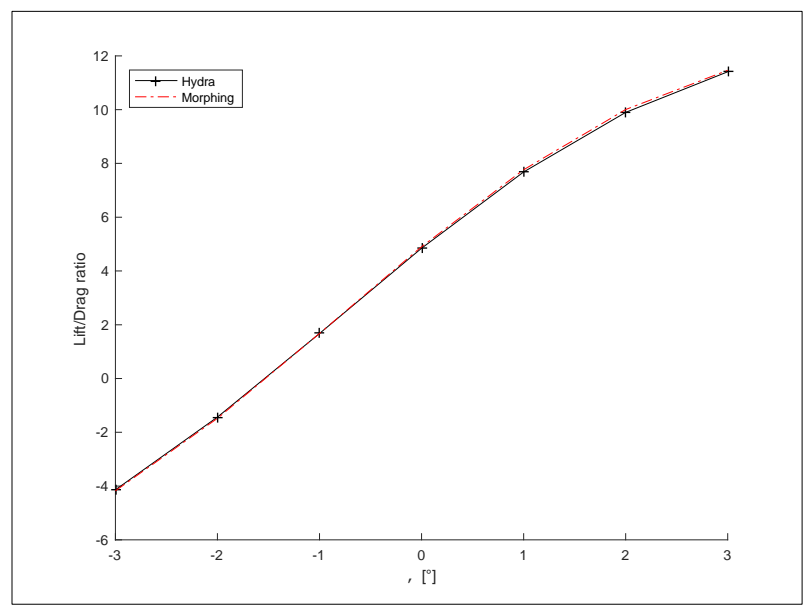

Figure 13: Lift over drag ratio comparison between Hydra foil and dynamic foil for Mach $=0.15$
In order to obtain the lift over drag ratio, the L/D was traced with respect to $\alpha$. For positive values of $\alpha$ 's, the Figure 13 shows that "morphing" curve is above "Hydra" curve. It means that morphing wing give better performances than the fixed wing.

Table 3 shows the percentage of lift over drag improvement with morphing wing, in a first sight the improvement is not linear. The negative sign of percentage of error notify an improvement but not for the $\alpha=-3^{\circ}$ value and the $\alpha=-2^{\circ}$ value because the negative sign is associated in these cases to the negative lift coefficient. Such as observations for Figure 13, over an angle of attack of 0 degrees the ratio is improved with $1 \%$. Below the angle of attack of 0 degrees the original airfoil is the best until -3 degrees. Because during the cruise an aircraft is flying with positive (or 0 degrees) angle of attack according to its weight, these last results can't affect the conclusion of the impact of the morphing wing during the cruise.

\begin{tabular}{|c|c|c|c|}
\hline$\alpha\left[^{\circ}\right]$ & $\mathrm{C}_{\mathrm{L}} / \mathrm{C}_{\mathrm{D}}$ Hydra & $\mathrm{C}_{\mathrm{L}} / \mathrm{C}_{\mathrm{D}}$ Morphing & \% error \\
\hline-3 & -4.14 & -4.18 & -0.87 \\
\hline-2 & -1.44 & -1.48 & -2.99 \\
\hline-1 & 1.67 & 1.68 & -0.09 \\
\hline 0 & 4.84 & 4.90 & -1.18 \\
\hline+1 & 7.68 & 7.77 & -1.11 \\
\hline+2 & 9.90 & 10.00 & -1.06 \\
\hline+3 & 11.41 & 11.46 & -0.52 \\
\hline
\end{tabular}

Table 3: Lift over Drag coefficients of original and morphed wing and for Mach number 0.15

\section{Conclusion}

In this paper, morphing wing benefits were found for the Hydra Technology UAS-S4 flying at cruise conditions. Optimized airfoils were positioned into a given section of the UAS-S4 wing. The wing was optimized especially for the reduction of drag coefficient $C_{D}$ for one constant Mach number. Although the efficiency of the morphing wing was proven with geometry details, the weight added to the wing with morphing wing system (actuators, sensors, etc) which allow to the airfoil to move according to the angle of attack during the flight was not taking into account. Because the lift force and the weight force are equal in cruise stage [11], the lift is going to be affected and consequently aerodynamics polar and Lift over drag ratio too.

To conclude this study, the morphing wing technology shown an improvement of $1 \%$ for the UAS-S4 when the geometry of its wing is only considered. Because the morphing wing is an hopeful technology and give some good results, some research have to lead in the future and a good compromise between weight of the morphing system and aerodynamic improvement associated have to be found to improve the entire aerodynamic of an aircraft. 


\section{Acknowledgement(s)}

We would like to thank the Hydra Technologies team in Mexico for their continuous support, especially to Mr. Carlos Ruiz, Mr. Eduardo Yakin and Mr. Alvaro Gutierrez Prado. We would also like to thank to the Natural Sciences and Engineering Research Council of Canada (NSERC) for the funding of the Canada Research Chair level 1 in Aircraft Modeling and Simulation [1] S. Kota, R. Osborn, G. Ervin, D. Maric, P. Flick, and D. Paul, "Mission adaptive compliant wing-design, fabrication and flight test," in RTO Applied Vehicle Technology Panel (AVT) Symposium, 2009.

[2] B. J. Maclean, B. F. Carpenter, and M. S. Misra, "Adaptive control surface using antagonistic shape memory alloy tendons," ed: Google Patents, 1997.

[3] J. D. Bartley-Cho, D. P. Wang, C. A. Martin, J. N. Kudva, and M. N. West, "Development of high-rate, adaptive trailing edge control surface for the smart wing phase 2 wind tunnel model," Journal of Intelligent Material Systems and Structures, vol. 15, pp. 279-291, 2004.

[4] J. N. Kudva, "Overview of the DARPA smart wing project," Journal of Intelligent Material Systems and Structures, vol. 15, pp. 261-267, 2004.

[5] M. Ghommem, N. Collier, A. Niemi, and V. M. Calo, "Shape optimization and performance analysis of flapping wings," in Proceedings of The Eighth International Conference on Engineering Computational Technology, 2012.

[6] A. Koreanschi, O. Sugar-Gabor, and R. Botez, "Drag optimisation of a wing equipped with a morphing upper surface," The Aeronautical Journal, vol. 120, pp. 473-493, 2016. [7] O. Ş. Gabor, A. Koreaschi, R. M. Botez, M. Mamou, and Y. Mebarki, "Analysis of the Aerodynamic Performance of a Morphing Wing-Tip Demonstrator Using a Novel Nonlinear Vortex Lattice Method," 2016.

[8] O. Sugar Gabor, A. Simon, A. Koreanschi, and R. M. Botez, "Application of a Morphing Wing Technology on Hydra Technologies Unmanned Aerial System UAS-S4," in The ASME 2014 International Mechanical Engineering Congress \& Exposition, Montreal, Que., Canada, 2014.

[9] A. Koreanschi, O. Ş. Gabor, T. Ayrault, R. M. Botez, M. Mamou, and Y. Mebarki, "Numerical Optimization and Experimental Testing of a Morphing Wing with Aileron System," in 24th AIAA/AHS Adaptive Structures Conference, 2016, p. 1083.

[10] A. A. de Paula, F. de Magalhães Porto, M. S. de Sousa, and S. S. da Cunha Junior, "Aerodynamic Longitudinal Coefficient Modeling During Aircraft Design Phases," in AIAA Modeling and Simulation Technologies Conference, 2016, p. 4133.

[11] G. Ghazi, "Développement d'une plateforme de simulation et d'un pilote automatique-application aux Cessna Citation X et Hawker 800XP," Master University of QuebecÉcole Polytechnique de Montréal, 2014.

[12] T. Grigorie, R. Botez, and A. Popov, "Design and experimental validation of a control system for a morphing wing," in AIAA Atmospheric flight mechanics conference, 2012.

[13] A. V. Popov, L. T. Grigorie, R. M. Botez, M. Mamou, and Y. Mébarki, "Real time morphing wing optimization validation using wind-tunnel tests," Journal of Aircraft, vol. 47, pp. 1346-1355, 2010.

[14] A. V. Popov, L. T. Grigorie, R. M. Botez, M. Mamou, and Y. Mebarki, "Closed-loop control validation of a morphing
Technologies. In addition, we would like to thank to the Canada Foundation of Innovation (CFI), to the Ministère du Développement économique, de l'Innovation et de l'Exportation (MDEIE) and to Hydra Technologies for the acquisition of the UAS-S4 using the Leaders Opportunity Funds.

\section{References}

wing using wind tunnel tests," Journal of Aircraft, vol. 47, pp. 1309-1317, 2010.

[15] O. Ş. Gabor, A. Koreanschi, and R. M. Botez, "A new non-linear vortex lattice method: Applications to wing aerodynamic optimizations," Chinese Journal of Aeronautics, vol. 29, pp. 1178-1195, 2016.

[16] O. S. Gabor, A. Koreanschi, and R. M. Botez, "Lowspeed aerodynamic characteristics improvement of ATR 42 airfoil using a morphing wing approach," in IECON 2012-38th Annual Conference on IEEE Industrial Electronics Society, 2012, pp. 5451-5456.

[17] O. S. Gabor, A. Simon, A. Koreanschi, and R. M. Botez, "Aerodynamic performance improvement of the UAS-S4 Éhecatl morphing airfoil using novel optimization techniques," Proceedings of the Institution of Mechanical Engineers, Part G: Journal of Aerospace Engineering, vol. 230, pp. 1164-1180, 2016.

[18] R. J. Margason and J. E. Lamar, Vortex-lattice FORTRAN program for estimating subsonic aerodynamic characteristics of complex planforms: National Aeronautics and Space Administration, 1971.

[19] T. Derbyshire and K. W. Sidwell, "PAN AIR summary document (version 1.0)," 1982.

[20] P. Baruah, J. Bussoletti, D. Chiang, W. Massena, F. Nelson, D. Furdon, et al., "PAN AIR: A computer program for predicting subsonic or supersonic linear potential flows about arbitrary configurations using a higher order panel method. Volume 4: Maintenance document (version 1.1)," 1981.

[21] M. S. Attia, D. E. Stevens, J. Vizcaino, and C. Tate, "2D and 3D Performance Prediction of a Convergent Nozzle at Pressure Ratios from 1.4 to 7 and Comparison with Wind Tunnel Data," in 49th AIAA/ASME/SAE/ASEE Joint PropulsionConference, ed, 2013, p. 3732.

[22] S. G. Hedman, "Vortex lattice method for calculation of quasi steady state loadings on thin elastic wings in subsonic flow," DTIC Document1966.

[23] J. Rom, B. Melamed, and D. Almosnino, "Experimental and nonlinear vortex lattice method results for variouswing-canard configurations," Journal of Aircraft, vol. 30, pp. 207-212, 1993.

[24] T. Melin, "A vortex lattice MATLAB implementation for linear aerodynamic wing applications," Master's Thesis, Department of Aeronautics, Royal Institute of Technology (KTH), Stockholm, Sweden, 2000. 